\title{
Effect of metformin and Pioglitazone on insulin dose reduction in type 2 diabetes mellitus patients: An open level comparative prospective study
}

\author{
Goutameswar Mazumdar $^{1^{*}}$, Bikash Swaika $^{2}$, Anindya Dasgupta $^{3}$ \\ ${ }^{1}$ Department of Pharmacology, Burdwan Medical College, Burdwan, India; ${ }^{*}$ Corresponding Author: goutameswar09@gmail.com \\ ${ }^{2}$ Department of General Medicine, Burdwan Medical College, West Bengal, India; dr.b.swaika@gmail.com \\ ${ }^{3}$ Department of Biochemistry, Burdwan Medical College, West Bengal, India; anindya11@yahoo.com
}

Received 1 October 2011; revised 4 November 2011; accepted 16 November 2011

\begin{abstract}
Insulin therapy cause weight gain and may increase its own requirement. Type 2 diabetes mellitus is associated with insulin resistance and affect both endogenous and exogenous insulin effect. Metformin and Pioglitazone, commonly used insulin sensitizers, have the potentiality of reducing the insulin dose, in Type 2 diabetes mellitus (T2DM) patients. This study was held to assess and compare such potentiality. 40 T2DM patients not controlled by diet, exercise and oral antidiabetic agents were selected for this study. Study had observed that Pioglitazone and metformin both significantly had reduced the dose of insulin. But metformin reduce the dose of insulin significantly more than that of Pioglitazone. Metformin had reduced the triglyceride, LDL-c, waist hip ratio significantly but modestly increased serum lactic acid and HDL-c. It can be concluded that co-administration of metformin with insulin is more beneficial in relation to the dose of insulin, waist hip ratio, and lipid modification when compare to Pioglitazone.
\end{abstract}

Keywords: Type 2 Diabetes Mellitus; Insulin Dose; Pioglitazone; Metformin

\section{INTRODUCTION}

Isolation of insulin represents one of the greatest medical discoveries [1]. Insulin is arguably still the mainstay of therapy for most people with type 2 diabetes mellitus (T2DM) [2] and particularly indicated who remain hyperglycemic with oral anti diabetic agents, diet, and exercise [3]. Insulin resistance is an impaired biologic response to either exogenously administered or endogenously secreted insulin [2] and is a consistent finding in patients with T2DM, and is present years before the onset of diabetes [4]. Insulin deficiency in T2DM is progressive and the likelihood that a patient will require insulin and continues to increase over time [5]. Weight gain in T2DM has been documented with insulin use [6] and become greater than those managed with other therapies [7]. Obesity is associated with decreased responsiveness to insulin in muscle, liver and fat [8] and weight gain of insulin therapy need more insulin and a potentially greater weight gain. Elevated concentration of insulin can cause insulin resistance by down regulating insulin receptors and desensitizing post receptor pathways [9]. Insulin plus an oral agent has shown equivalent to or better than insulin only regimens in some circumstances [10]. Pioglitazone and metformin are insulin sensitizers and improve insulin resistance inT2DM. Adding metformin to insulin for patients with poorly controlled T2DM lowers glucose and lipid levels more effectively than increasing insulin dose alone [11] and addition of thiazolidinediones significantly improved the glycemic control and followed for a significant reduction of daily insulin requirements [12].

Therefore a study has been designed with the aim to compare the insulin dose reducing capability of metformin and Pioglitazone in T2DM patients. The objective of this study is to assess the requirement of insulin dose for glycemic control when combined with Pioglitazone and metformin individually and sequentially.

\section{MATERIALS AND METHODS}

This study was conducted in the medical outpatient department, department of biochemistry and pharmacology of Burdwan Medical College for a period of six months. 40 T2DM patients, uncontrolled by diet, exercise, and oral anti diabetic agents in adequate dose and duration had been selected for this study. This study was conducted according to the Helsinki dceclaration 1975 
and ICMR guideline. Exclusion criteria was unable to give informed consent, renal impairment, hepatic disease, heart failure, lactic acidosis, alcoholics, pregnancy, female subjects planning for pregnancy, subjects having chronic hypoxic lung disease, taking low calorie diet and $\mathrm{h} / \mathrm{o}$ long standing repeated fasting. This study was approved by the institutional ethics committee. Written informed consent was taken from all the study subjects. The study design was open level self controlled sequential interventional prospective study. Base line fbg and ppbg and Hbalc were measured at the time of patient selection. Premixed insulin was started at a dose of 0.5 $\mathrm{iu} / \mathrm{kg}$ body weight in two divided doses $\left(2 / 3^{\text {rd }}\right.$ pre breakfast and $1 / 3^{\text {rd }}$ pre diner dose). Blood glucose was measured after one week interval. Increment of insulin dose was done by 4 units every week (in two divided doses) till blood glucose comes down to near normal. Then Pioglitazone was started at a dose of $15 \mathrm{mg} / \mathrm{d}$ initially. After a period of 4 weeks, Pioglitazone dose was escalated to $30 \mathrm{mg} / \mathrm{d}$. Dose of insulin was decreased gradually and proportionately in each step keeping blood glucose near normal. Then after a period of 4 weeks blood glucose was measured.
Pioglitazone was then withdrawn and keeping the subjects Pioglitazone free for one week (washout period) and dose of insulin at this time was kept at the previous base line dose. Then metformin was initiated with dose of $850 \mathrm{mg} /$ day and then the dose of metformin gradually increased up to $850 \mathrm{mg}$ three times per day $(2550$ $\mathrm{mg} /$ day) in a period of two weeks. Proportionately dose of insulin was also decreased keeping blood glucose near normal and maintained it further for a period of 4 weeks. Insulin dose in all these steps was noted.

Statistical methods: Data had presented as mean with standard deviation. Paired sample " $t$ " test was employed to test the significance of insulin dose reducing effect within the same study cohort group. For all analyses p-value $<0.05$ was considered as statistically significant. Statistical analysis was performed by using spss-17 software.

\section{RESULTS}

40 uncontrolled T2DM pts had received insulin, insulin + Pioglitazone, insulin+metformin sequentially over a period of six months and had completed this study (See Tables 1-2).

Table 1. Demographic profile of the study subjects.

\begin{tabular}{|c|c|c|c|c|c|c|c|c|c|c|c|}
\hline $\begin{array}{c}\text { No of } \\
\text { pts }\end{array}$ & $\begin{array}{l}\text { Mean age } \\
\text { (SD) In yrs }\end{array}$ & sex & religion & $\begin{array}{l}\text { Mean B wt } \\
\text { In Kg (SD) }\end{array}$ & $\begin{array}{c}\text { Per capita } \\
\text { monthly } \\
\text { income (Rs) }\end{array}$ & $\begin{array}{c}\text { Mean duration } \\
\text { of diabetes } \\
\text { In yrs }\end{array}$ & $\begin{array}{c}\text { Family H/O } \\
\text { of diabetes } \\
\text { In yrs }\end{array}$ & Education & Occupation & Residence & $\begin{array}{l}\text { Mean BMI } \\
\text { (SD) } \mathrm{Kg} / \mathrm{m}^{2}\end{array}$ \\
\hline
\end{tabular}

Note: no-number, pts-patients, SD-standard deviation, Bwt-body weight, m-male, f-female, h-Hindu, mu-Muslim, HW-house wife, ser-service, H/O-history of, BMI-body mass index, $\mathrm{kg} / \mathrm{m}^{2}$-kilogram/meter square. Study subjects were predominantly male $(62.5 \%)$, elderly (48.65 \pm 12.13 yrs), non obese (BMI $21.25 \pm$ $4.04 \mathrm{~kg} / \mathrm{m}^{2}$ ), poor (Rs $1034 /$-per month) patients with positive family history of T2DM $45 \%$.

Table 2. Change of value from baseline after therapy with insulin, insulin + Pioglitazone, insulin + metformin given sequentially.

\begin{tabular}{ccccc}
\hline character & Base line (mean \pm SD) & Insulin (mean \pm SD) & Insulin + Pioglitazone (mean \pm SD) & Insulin + metformin $($ mean \pm SD) \\
\hline Fbg (mg/dl) SD & $279.7 \pm 55.85$ & $102.67 \pm 22.67$ & $104.12 \pm 4.42$ & $101.15 \pm 4.27^{*}$ \\
Ppbg (mg/dl) SD & $376.52 \pm 88.13$ & $169.07 \pm 9.32$ & $170.02 \pm 7.51$ & $168.17 \pm 8.99$ \\
HbA1c (\%) & $11.36 \pm 0.9020$ & $6.24 \pm 1.0755$ & $6.11 \pm 0.5313$ & $6.06 \pm 0.5346$ \\
W/H ratio & $0.958 \pm 0.0788$ & $0.971 \pm 0.0753$ & $0.971 \pm 0.0736$ & $0.954^{* *} \pm 0.0811$ \\
TGL (mg/dl) & $186.6 \pm 48.0276$ & $173.85 \pm 41.3469$ & $182.35 \pm 40.8509$ & $164.47^{*} \pm 37.1379$ \\
HDL-c (mg/dl) & $50.1 \pm 6.3329$ & $51.62 \pm 7.1456$ & $47.92 \pm 6.5491$ & $54.2^{*} \pm 7.6600$ \\
LDL-c (mg/dl) & $125.1 \pm 40.0101$ & $117.92 \pm 37.2925$ & $124.07 \pm 37.6334$ & $110.55^{*} \pm 34.5230$ \\
SGPT (iu/l) & $22.52 \pm 5.2622$ & $22.47 \pm 5.301$ & $22.25 \pm 7.9103$ & $17.8 \pm 5.44$ \\
S.Lactic acid (mg/dl) & $6.45 \pm 0.7128$ & $6.47 \pm 0.7065$ & $6.39 \pm 0.6053$ & $6.76^{*} \pm 0.6306$ \\
Dose of insulin(u) SD & & $29.3 \pm 7.0463$ & $25.725 \pm 6.47$ & $22.87 \pm 6.03^{* *}$ \\
\hline
\end{tabular}

Note: fbg-fasting blood glucose, mg/dl-milligram/deciliter, SD-standard deviation, bA1c-glycosylated hemoglobin, W/H-waist/hip, TGL-triglyceride, HDL-cigh density cholesterol, LDL-c-low density cholesterol, SGPT(iu/l)-serum glutamic pyruvate transaminase (international unit/liter), ${ }^{*}:$ significant, ${ }^{* *}:$ highly significant. 
Insulin had reduced the blood glucose from base line. Addition Pioglitazone and metformin maintain blood glucose nearly same level but at significantly $(\mathrm{p}<0.001)$ low dose of insulin (mean reduction of insulin dose for Pioglitazone and metformin $3.57 \pm 1.106 \mathrm{u} / \mathrm{d}$ and $6.425 \pm$ $1.737 \mathrm{u} / \mathrm{d}$ respectively with $95 \%$ CI $3.221-3.928$ and 5.869 - 6.980 respectively). Insulin metformin combination cause significantly $(p<0.001)$ more reduction of insulin dose requirement than insulin Pioglitazone combination (mean difference $2.850 \pm 1.1886 \mathrm{u} / \mathrm{d}, 95 \%$ CI 2.469 - 3.230, t-15.163).

Waist hip ratio is significantly $(0.001)$ reduced with metformin combination than that of Pioglitazone combination $(95 \% \mathrm{CI}-0.034$ to -0.011$)$ and Pearson correlation was 0.896 . Triglyceride and LDL-cholesterol was reduced in both Pioglitazone and metformin combination but significantly more with metformin. HDL-cholesterol decreased with Pioglitazone but increased with metformin.

Serum lactic acid decreased and SGPT increased with Pioglitazone and opposite effect observed with metformin but modest.

\section{DISCUSSION}

Because of progressive deficiency of insulin [5], exogenous insulin administration may require at some point of time inT2DM and Insulin resistance further enhance the requirement to make the subjects euglycemic. Insulin sensitizers improve insulin resistance and reduce the insulin dose. To minimize the insulin dose and risk it may entail, insulin therapy should be combined with other measures including oral hypoglycemic drugs [13]. Metformin and Pioglitazone are commonly used insulin sensitizers, and Premixed 30/70 insulin is the commonly used simple and may be the best option for the $t 2 \mathrm{dm}$ patients [5], and had used in this study. Pioglitazone and metformin administration along with insulin individually reduced the insulin dose significantly $(p<0.001)$. In fact addition of metformin or thiazolidinediones to insulin for patients with poorly controlled type 2 diabetes improve the glycemic control and reduce daily insulin requirements $[11,12]$. Study had observed that metformin reduced the dose of insulin significantly $(p<0.001)$ more than Pioglitazone (mean reduction of dose $2.85 \pm 1.118$ $\mathrm{u} / \mathrm{d}$ ). Nausea, upper abdomen distension, flatulence, diarrhea are the common tolerable side effects of metformin, therefore metformin the dose of metformin increased gradually over weeks. The response of Pioglitazone comes after weeks to month, to observe the response dose escalation of Pioglitazone was done after 4 weeks.

Metformin suppress hepatic gluconeogenesis, reduce the effect of glucagon, and promote the insulin and glu- cose sensitive transport properties of glucose transporters [14], reduce body weight and insulin resistance. Pioglitazone activate the PPAR-Y receptor, reduced insulin resistance, decrease the release of free fatty acids and cytokines, increase adiponectin, augment glucose transport in muscle, raise glycogen synthesis in liver [15]. Weight gain is associated with insulin use and proposed explanations are 1) decrease glycosuria so decrease weight loss; 2) direct lipogenic effects of insulin 3) recurrent mild hypoglycemia leads to hunger so increase caloric intakes [16]. Co administration of Pioglitazone and met individually with insulin significantly reduce insulin dose. But met decrease body weight and Pioglitazone increase it. Study observed metformin decrease insulin dose significantly more than that of Pioglitazone. But metformin decreased waist: hip ratio, triglyceride, LDL-cholesterol and increased HDL-cholesterol significantly more than Pioglitazone. Therefore it is lipid friendly.

\section{REFERENCES}

[1] Banting, F.M. and Best, C.H. (1922) The internal secretion of the pancreas. Journal of Laboratory and Clinical Medicine, 7, 256-271

[2] Buse, J.B., Polonsky, K.S. and Burant, C.F. (2008) Type 2 diabetes mellitus. In: Kronenberg, H.M., Melmed, S., Polanski, K.S. and Larsen, P.R., Eds., Williams Textbook of Endocrinology, 11th Edition, Saunders Elsevier, Philadelphia, 1329-1389.

[3] Yki-Javimen, H., Kaupilla, M., Kujansuu, E., et al. (1992) Comparison of insulin regimens in patients with non-insulin-dependent diabetes mellitus. New England Journal of Medicine, 327, 1426-1433. doi:10.1056/NEJM199211123272005

[4] Warram, J.H., Martin, B.C., Krowelski, A.S., et al. (1990) Slow glucose removal and hyperinsulinemia precede the development of type 2 diabetes in the offspring of diabetic parents. Annals of Internal Medicine, 113, 909-915.

[5] Cheng, Y.Y.A. and Zinman, B. (2010) Principles of insulin therapy. In: Khan, C.R., Weir, G.C., King, G.L., Jacobson, A.M., Moses, A.C. and Smith, R.J., Eds. Joslin's Diabetes Mellitus, 14th Edition, Wolters Kluwer (India) Pvt Ltd., New Delhi, 659-670.

[6] The Diabetes Control and Complications Research Group (1995) Adverse events and their association with treatment regimens in the diabetes control and complications trial. Diabetes Care, 18, 1415-1427.

doi:10.2337/diacare.18.11.1415

[7] UKPDS Group (1998) Intensive blood glucose control with sulphonulureasor insulin compared with conventional treatment and risk of complication in patients with type 2 diabetes (UKPDS 33). Lancet, 352, 837-853.

[8] Field, J.B. (1970) Chronic insuli resistance. Acta Diabetologica, 7, 220-242. doi:10.1007/BF01556784

[9] Inoguchi, T., Xia, P., Kunisaki, M., et al. (1994) insulin 
effect on protein kinase $\mathrm{C}$ and diacylglycerol induced by diabetes and glucose in vascular tissues. American Journal of Physiology, 267, E369-E379.

[10] Fonseca, V., Graveline, J. and Nissel, J. (1998) Long term experience with troglitazone in combination with insulin type 2 diabetes mellitus. Diabetes, 47, A90 (abt).

[11] Relimpio, F., Pumer, A., Losado, F., et al. (1998) Adding metformin versus insulin dose increase in insulin treated but poorly controlled type 2 diabetes mellitus. An open label randomized trial. Diabetic Medicine, 15, 997-1002.

[12] Schwartz, S., Raskin, P., Fonsec, V., et al. (1998) Effect of troglitazone in insulin-treated patients with type 2 diabetes mellitus. New England Journal of Medicine, 338,
861-866. doi:10.1056/NEJM199803263381302

[13] Henry, R.R. (1996) Glucose control and insulin resistance in non-insulin dependent diabetes mellitus. Annals of Internal Medicine, 124, 97-103.

[14] Bailey, C.J. Metformin in therapy for diabetes mellitus and related disorders. 5th Edition, ADA, 235-253.

[15] Lebovitz, H.E. Thiazolidinedione in therapy for diabetes mellitus and related disorders. 5th Edition, ADA, 260272.

[16] Torbay, N., Bracco, E., Geliebter, A., et al. (1985) Insulin increases body fat despite control of food intake and physical activity. American Journal of Physical, 258, R2120R2144. 